\title{
Polarimetric Formulation of the Visibility Function Equation Including Cross-Polar Antenna Patterns
}

\author{
Adriano Camps, Senior Member, IEEE, Ignasi Corbella, Member, IEEE, Francesc Torres, Member, IEEE, \\ Mercè Vall-llossera, Member, IEEE, and Nuria Duffo, Member, IEEE
}

\begin{abstract}
The European Space Agency's Soil Moisture and Ocean Salinity (SMOS) mission will be the first one using two-dimensional aperture synthesis radiometry for earth observation. This study presents the formulation that relates instrument observables and brightness temperature maps including cross-polar antenna voltage patterns, which may be also different from element to element. Finally, the radiometric accuracy degradation if cross-polar patterns are neglected in the image reconstruction is studied.
\end{abstract}

Index Terms-Antenna arrays, interferometry, radiometry.

\section{INTRODUCTION}

$\mathbf{T}$ HE Microwave Imaging Radiometer by Aperture Synthesis (MIRAS) instrument [1], [2] is the single payload of the European Space Agency's Soil Moisture and Ocean Salinity (SMOS) mission, and it will be the first two-dimensional aperture synthesis radiometer for earth observation. The brightness temperature image reconstruction is performed through a Fourier synthesis process of the cross-correlations $V_{12}^{p q}$ measured between the band pass signals $b_{1}(t)$ and $b_{2}(t)$ -centered at $f_{0}, \lambda_{0}=c / f_{0}$ - collected by every pair of elements in the array located in the $X Y$ plane. According to [3]

$$
\begin{aligned}
V_{12}^{p q} \triangleq & \frac{1}{k_{B} \sqrt{B_{1} B_{2}} \sqrt{G_{1} G_{2}}} \cdot \frac{1}{2}\left\langle b_{1}^{p}(t) b_{2}^{q^{*}}(t)\right\rangle \\
= & \frac{1}{\sqrt{\Omega_{1} \Omega_{2}}} \iint_{\xi^{2}+\eta^{2} \leq 1} \frac{\left(T_{p q}(\xi, \eta)-T_{\mathrm{rec}} \delta_{p q}\right)}{\sqrt{1-\xi^{2}-\eta^{2}}} \\
& \cdot F_{n p 1}(\xi, \eta) F_{n q 2}^{*}(\xi, \eta) \tilde{r}_{12}\left(-\frac{u_{12} \xi+v_{12} \eta}{f_{0}}\right) \\
& \cdot \exp \left(-j 2 \pi\left(u_{12} \xi+v_{12} \eta\right)\right) d \xi d \eta
\end{aligned}
$$

where $k_{B}$ is the Boltzmann's constant, $B_{1,2}$ and $G_{1,2}$ are the receivers' noise bandwidth and power gain, $\Omega_{1,2}$ is the solid angle of the antennas, $T_{p q}(\xi, \eta)$ is the brightness temperature of the scene [see (4)], $T_{\text {rec }}$ is the physical temperature of the receivers [3], $\delta_{p q}=1$ if $p=q$ and 0 if $p \neq q$,

Manuscript received October 10, 2003; revised February 4, 2004. This work was supported in part by the Spanish Comisión Interministerial de Ciencia y Tecnología under Grant MCYT TIC2002-04451-C02-01 and in part by EADSCASA Space Division (Madrid, Spain), prime contractor of SMOS PLM during Phase B Activities.

The authors are with the Universitat Politècnica de Catalunya, E-08034 Barcelona, Spain (e-mail: camps@tsc.upc.es).

Digital Object Identifier 10.1109/LGRS.2005.846885
$F_{n p, q 1,2}(\xi, \eta)$ are the normalized antenna copolar voltage patterns at $p$ and $q$ polarizations, $\tilde{r}_{12}\left(-\left(u_{12} \xi+v_{12} \eta\right) / f_{0}\right)$ is the fringe-washing function, $\left(u_{12}, v_{12}\right)$ is the spatial frequency (baseline) that depends on the antenna position difference: $\left(u_{12}, v_{12}\right)=\left(x_{2}-x_{1}, y_{2}-y_{1}\right) / \lambda_{0}$, and the director cosines $(\xi, \eta)=(\sin \theta \cos \phi, \sin \theta \sin \phi)$ are defined with respect to the $X$ and $Y$ axes.

\section{Brightness Temperature Measured by the INTERFEROMETRIC RADIOMETER: IDEAL CASE With ISOTROPIC ANTENNA VOLTAGE PATTERNS AND ZERo CROSS-POLAR PATTERNS}

Due to the following:

- the relative orientation $[\psi(\xi, \eta)]$ between the pixel's reference frame over the earth's surface ( $V$ or $H$ : vertical and horizontal polarizations) and the antenna reference frame [4] ( $X$ is perpendicular to the orbital plane; $Y$ is $32^{\circ}$ upward titled from the velocity vector);

- Faraday rotation effects $[\phi(\xi, \eta)]$

the electric fields incident in the antennas are rotated an angle $\alpha(\xi, \eta)=\psi(\xi, \eta)+\phi(\xi, \eta)[4]$

$$
\left[\begin{array}{l}
E_{x} \\
E_{y}
\end{array}\right]=\left[\begin{array}{cc}
A & B \\
-B & A
\end{array}\right]\left[\begin{array}{l}
E_{h} \\
E_{v}
\end{array}\right]
$$

where $A=\cos (\alpha), B=\sin (\alpha)$. Therefore, since $T_{p q}(\xi, \eta)$ is proportional to $\left\langle E_{p} E_{q}^{*}\right\rangle / 2$, the brightness temperatures in the antenna reference frame at a given polarization $(X$ or $Y)$ can be expressed as a linear combination of the brightness temperatures in the earth's pixel reference frame ( $H$ or $V)$ [5, eq. (57)].

$$
\left[\begin{array}{c}
T_{x x} \\
T_{x y} \\
T_{y x} \\
T_{y y}
\end{array}\right]=\left[\begin{array}{cccc}
A^{2} & A B & A B & B^{2} \\
-A B & A^{2} & -B^{2} & A B \\
-A B & -B^{2} & A^{2} & A B \\
B^{2} & -A B & -A B & A^{2}
\end{array}\right]\left[\begin{array}{l}
T_{h h} \\
T_{h v} \\
T_{v h} \\
T_{v v}
\end{array}\right]
$$

where $T_{v h}=T_{h v}^{*} \hat{=}(U+j V) / 2$, and $U$ and $V$ are the third and fourth Stokes parameters in the earth's reference frame. Compared to $T_{h h}$ and $T_{v v}, U$ and $V$ have negligible values, and even more at L-band. Therefore, $\operatorname{Im}\left(T_{x y}\right)=\operatorname{Im}\left(T_{y x}\right)=0$ and $\operatorname{Re}\left(T_{x y}\right)=\operatorname{Re}\left(T_{y x}\right)$, and $T_{x y}=T_{y x}=\operatorname{Re}\left(T_{x y}\right)=\operatorname{Re}\left(T_{y x}\right)$. Equation (3) reduces to

$$
\left[\begin{array}{l}
T_{x x} \\
T_{y x} \\
T_{y y}
\end{array}\right]=\left[\begin{array}{cc}
A^{2} & B^{2} \\
-A B & A B \\
B^{2} & A^{2}
\end{array}\right]\left[\begin{array}{l}
T_{h h} \\
T_{v v}
\end{array}\right] .
$$



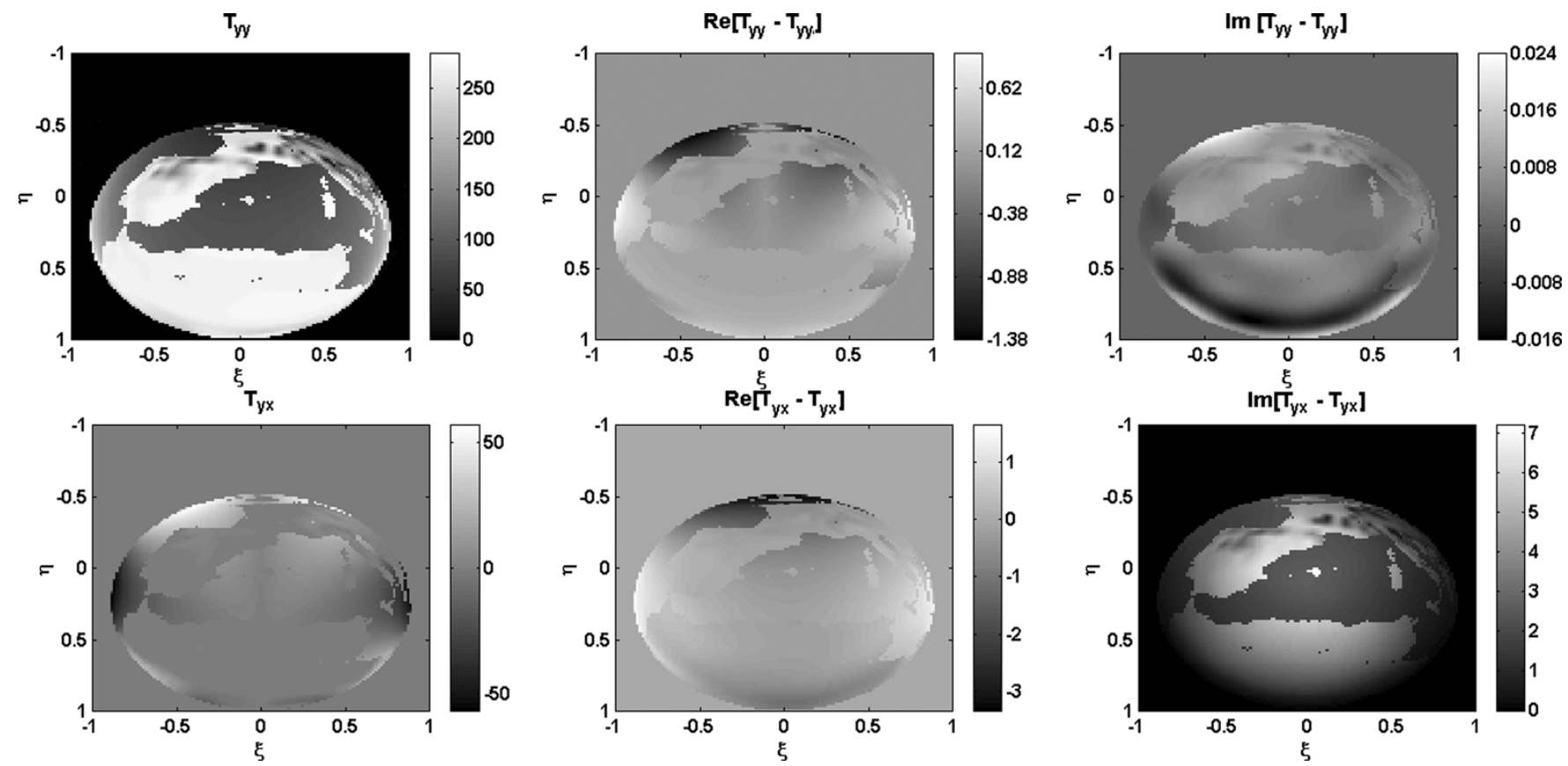

Fig. 1. Image errors maps in a high-contrast scene if antenna cross-polarization patterns are neglected: $T_{y y}=A^{2} T_{v v}+B^{2} T_{h h}$ and $T_{y x}=A B\left(T_{v v}-T_{h h}\right)$. Root mean squared errors in the alias-free field of view: $\sigma_{\operatorname{Re}\left[\hat{T}_{x x}^{i d}-\hat{T}_{x x}^{i d}\right]}=0.18 \mathrm{~K}, \sigma_{\operatorname{Im}\left[\hat{T}_{x x}-\hat{T}_{x x}^{i d}\right]}=5.510^{-3} \mathrm{~K}, \sigma_{\operatorname{Re}\left[\hat{T}_{y y}-\hat{T}_{y y}^{i d}\right]}=0.15 \mathrm{~K}, \sigma_{\operatorname{Im}\left[\hat{T}_{y y}-\hat{T}_{y y}^{i d}\right]}=$ $3.610^{-3} \mathrm{~K}, \sigma_{\operatorname{Re}\left[\hat{T}_{y x}-\hat{T}_{y x}^{i d}\right]}=0.30 \mathrm{~K}$ and $\sigma_{\operatorname{Im}\left[\hat{T}_{y x}-\hat{T}_{y x}^{i d}\right]}=3.5 \mathrm{~K}$.

\section{BRIGHTNESS TEMPERATURE MEASURED BY} THE INTERFEROMETRIC RADIOMETER: REAL CASE INCLUDING ANTENNA COPOLAR AND NONZERO CROSS-POLAR PATTERNS

If nonzero cross-polar antenna patterns are taken into account, a similar process to the one followed to derive (2)-(4), can be used to derive the cross-brightness temperatures to be used in (1)

$$
\left[\begin{array}{l}
\hat{E}_{x 1,2} \\
\hat{E}_{y 1,2}
\end{array}\right]=\left[\begin{array}{ll}
R_{x 1,2} & C_{x 1,2} \\
C_{y 1,2} & R_{y 1,2}
\end{array}\right]\left[\begin{array}{c}
E_{x 1,2} \\
E_{y 1,2}
\end{array}\right]
$$

where the symbol $\hat{E}_{x, y 1,2}$ denotes the received electric field including the effect of the antenna voltage patterns, $R_{x, y 1,2}(\xi, \eta) \equiv F_{n x, y 1,2}(\xi, \eta)$ is the copolar pattern of antenna 1 or 2 , at $X$ or $Y$ polarization, and $C_{x, y 1,2}(\xi, \eta)$ is the cross-polar antenna pattern, both normalized to the maximum of $R_{x, y 1,2}(\xi, \eta)$, and defined according to Ludwig's third definition [6]. ${ }^{1}$ Expanding (5), the relationship between

${ }^{1}$ In (5) the measured co- and cross-polar patterns at $X$ - and $Y$-polarizations correspond to the two outputs of a dual-polarization antenna as normally measured. If the $Y$-pattern were obtained by a rotation of the $X$-pattern, then the cross-polar pattern $C_{y 1,2}$ must be replaced by $-C_{y 1,2}$.
$\hat{T}_{x x}, \hat{T}_{y y}$ and $\hat{T}_{y x}$ and $T_{x x}, T_{y y}$ and $T_{x y}$ can be readily obtained

$$
\hat{T}_{x x}=R_{x 1} R_{x 2}^{*} T_{x x}+\left(R_{x 1} C_{x 2}^{*}+C_{x 1} R_{x 2}^{*}\right) T_{y x}+C_{x 1} C_{x 2}^{*} T_{y y}
$$

$\hat{T}_{y y}=C_{y 1} C_{y 2}^{*} T_{x x}+\left(R_{y 1} C_{y 2}^{*}+C_{y 1} R_{y 2}^{*}\right) T_{y x}+R_{y 1} R_{y 2}^{*} T_{y y}$

$\hat{T}_{y x}=C_{y 1} R_{x 2}^{*} T_{x x}+\left(R_{y 1} R_{x 2}^{*}+C_{y 1} C_{x 2}^{*}\right) T_{y x}+R_{y 1} C_{x 2}^{*} T_{y y}$

The term " $T_{\text {rec }}$ " in (1) accounts for the thermal noise radiated by the receivers at both polarizations (but not at the cross-polar one) that is collected by other elements because of the nonzero antenna coupling. This noise is collected together with the noise coming from the scene being imaged. Therefore, following a similar procedure to the one described in previous paragraphs for $T_{p q}$, (1) should be modified for the $T_{\text {rec }}$ term accordingly

$$
\begin{aligned}
& \hat{T}_{\text {rec } x x}=R_{x 1} R_{x 2}^{*} T_{\text {rec }}+C_{x 1} C_{x 2}^{*} T_{\text {rec }} \\
& \hat{T}_{\text {rec } y y}=C_{y 1} R_{x 2}^{*} T_{\text {rec }}+R_{y 1} C_{x 2}^{*} T_{\text {rec }} \\
& \hat{T}_{\text {rec } y x}=0 .
\end{aligned}
$$

Substituting (6)-(9) in (1), (1) can be rewritten making the following substitutions, as in (10), shown at the bottom of the page, where it has been assumed that $T_{y x}=T_{x y}$.

$$
\begin{gathered}
\left(T_{x x}(\xi, \eta)-T_{\mathrm{rec}}\right) F_{n x 1}(\xi, \eta) F_{n x 2}^{*}(\xi, \eta) \rightarrow R_{x 1} R_{x 2}^{*}\left(T_{x x}-T_{\mathrm{rec}}\right)+\left(R_{x 1} C_{x 2}^{*}+C_{x 1} R_{x 2}^{*}\right) T_{y x}+C_{x 1} C_{x 2}^{*}\left(T_{y y}-T_{\mathrm{rec}}\right), \\
\left(T_{y y}(\xi, \eta)-T_{\mathrm{rec}}\right) F_{n y 1}(\xi, \eta) F_{n y 2}^{*}(\xi, \eta) \rightarrow C_{y 1} C_{y 2}^{*}\left(T_{x x}-T_{\mathrm{rec}}\right)+\left(R_{y 1} C_{y 2}^{*}+C_{y 1} R_{y 2}^{*}\right) T_{y x}+R_{y 1} R_{y 2}^{*}\left(T_{y y}-T_{\mathrm{rec}}\right), \\
T_{y x}(\xi, \eta) F_{n y 1}(\xi, \eta) F_{n x 2}^{*}(\xi, \eta) \rightarrow C_{y 1} R_{x 2}^{*}\left(T_{x x}-T_{\mathrm{rec}}\right)+\left(R_{y 1} R_{x 2}^{*}+C_{y 1} C_{x 2}^{*}\right) T_{y x}+R_{y 1} C_{x 2}^{*}\left(T_{y y}-T_{\mathrm{rec}}\right)
\end{gathered}
$$




\section{ERRORS ASSOCIATED TO NONZERO CROSS-POLAR ANTENNA PATTERNS IN THE IMAGE RECONSTRUCTION ALGORITHMS IN THE ANTENNA REFERENCE FRAME}

In order to study the impact of neglecting antenna cross-polar pattern in the image reconstruction algorithms, synthetic brightness temperature images have been generated using the SMOS End-to-end Performance Simulator (SEPS) [7]. The co- and cross-polar antenna radiation voltage patterns used correspond to the first nine receiver units (LICEF-2) of MIRAS and exhibit a directivity of $9 \mathrm{~dB}$ approximately, with cross-polar level at boresight of $-30 \mathrm{~dB}$ approximately, and $-25 \mathrm{~dB}$ worst case at the border of the alias-free field of view, which is determined by the periodic repetition of six replicas of the earth's image [7].

Fig. 1 (top) shows the difference between $T_{y y}$ when the cross-polar component of the antenna voltage patterns is assumed to be zero, and (7) with nonzero cross-polar component, for a high-contrast scenario, including land and sea. Fig. 1 (bottom) presents a similar result for $T_{y x}$. Error images at $X$ polarization are very similar to the ones at $Y$ polarization, and are not shown. The rms errors computed in the alias-free field-of-view at different polarizations are: $\sigma_{\operatorname{Re}\left[\hat{T}_{x x}-\hat{T}_{x x}^{i d}\right]}=0.18 \mathrm{~K}, \sigma_{\operatorname{Im}\left[\hat{T}_{x x}-\hat{T}_{x x}^{i d}\right]}=5.510^{-3} \mathrm{~K}$, $\sigma_{\operatorname{Re}\left[\hat{T}_{y y}-\hat{T}_{y y}^{i d}\right]}=0.15 \mathrm{~K}, \sigma_{\operatorname{Im}\left[\hat{T}_{y y}-\hat{T}_{y y}^{i d}\right]}=3.610^{-3} \mathrm{~K}$, $\sigma_{\operatorname{Re}\left[\hat{T}_{y x}-\hat{T}_{y x}^{i d}\right]}=0.30 \mathrm{~K}$ and $\sigma_{\operatorname{Im}\left[\hat{T}_{y x}-\hat{T}_{y x}^{i d}\right]}=3.5 \mathrm{~K}$ where the superscript " $i d$ " refers to the $\hat{T}_{p q}$ if the cross-polar patterns were zero. It would be expected that the smaller the difference between $T_{y y}$ and $T_{x x}$, the smaller the effect of nonzero cross-polar pattern errors. However, a low-contrast only-ocean scene has similar errors at $X$ - and $Y$-polarizations, and just slightly smaller at $Y X$-polarization: $\sigma_{\operatorname{Re}\left[\hat{T}_{y x}-\hat{T}_{y x}^{i d]}\right]}=0.48 \mathrm{~K}$, and $\sigma_{\operatorname{Im}\left[\hat{T}_{y x}-\hat{T}_{y x}^{i d}\right]}=1.9 \mathrm{~K}$. Errors in the imaginary parts of $T_{x x}$ and $T_{y y}$ come from neglecting cross-polar antenna patterns in the image reconstruction. At the present time there is not an explanation for the fact that the error on $T_{y x}$ is found with the same sign throughout the field of view, but it is believed that it may be due to a phase offset between the $Y$ and $X$ antenna patterns, due to different path lengths from the antenna to the receivers' input.

\section{ERROR AMPLIFICATION IN THE TRANSFORMATION FrOM ANTENNA REFERENCE FRAME TO EARTH REFERENCE FRAME}

MIRAS has two operation modes.

- Full-polarimetric mode, in which $\hat{T}_{x x}, \hat{T}_{y y}$ and $\hat{T}_{x y}$ are measured by a sophisticated combination of the antenna polarization switches at $X$ and $Y$ polarizations [5], and

- Dual-polarization mode, in which $\hat{T}_{x x}$ and $\hat{T}_{y y}$ are sequentially measured, by selecting all the antenna polarization switches at $X$ and $Y$ polarizations simultaneously.

In the full-polarimetric mode, the brightness temperatures in the earth reference frame $\left(\hat{T}_{h h}, \hat{T}_{v v}, \hat{T}_{h v}\right.$, and $\left.\hat{T}_{v h}\right)$ are computed from the measured brightness temperatures in the antenna reference frame $\left(\hat{T}_{x x}, \hat{T}_{y y}, \hat{T}_{x y}\right.$ and $\left.\hat{T}_{y x}\right)$ by inverting (3)

$$
\left[\begin{array}{l}
\hat{T}_{h h} \\
\hat{T}_{h v} \\
\hat{T}_{v h} \\
\hat{T}_{v v}
\end{array}\right]=\left[\begin{array}{cccr}
A^{2} & -A B & -A B & B^{2} \\
A B & A^{2} & -B^{2} & -A B \\
A B & -B^{2} & A^{2} & -A B \\
B^{2} & A B & A B & A^{2}
\end{array}\right]\left[\begin{array}{l}
\hat{T}_{x x} \\
\hat{T}_{x y} \\
\hat{T}_{y x} \\
\hat{T}_{y y}
\end{array}\right]
$$

and errors in the antenna reference frame translate into errors in the earth reference frame

$$
\left[\begin{array}{l}
\Delta \hat{T}_{h h} \\
\Delta \hat{T}_{h v} \\
\Delta \hat{T}_{v h} \\
\Delta \hat{T}_{v v}
\end{array}\right]=\left[\begin{array}{cccr}
A^{2} & -A B & -A B & B^{2} \\
A B & A^{2} & -B^{2} & -A B \\
A B & -B^{2} & A^{2} & -A B \\
B^{2} & A B & A B & A^{2}
\end{array}\right]\left[\begin{array}{l}
\Delta \hat{T}_{x x} \\
\Delta \hat{T}_{x y} \\
\Delta \hat{T}_{y x} \\
\Delta \hat{T}_{y y}
\end{array}\right] .
$$

Note that the inverse of the matrix in (3) is equal to its transpose.

In the dual-polarization mode, the brightness temperatures in the earth reference frame $\left(\hat{T}_{h h}\right.$ and $\left.\hat{T}_{v v}\right)$ are computed from (4), assuming $T_{y x}=0$

$$
\left[\begin{array}{l}
\hat{T}_{h h} \\
\hat{T}_{v v}
\end{array}\right]=\frac{1}{A^{4}-B^{4}}\left[\begin{array}{rr}
A^{2} & -B^{2} \\
-B^{2} & A^{2}
\end{array}\right]\left[\begin{array}{l}
\hat{T}_{x x} \\
\hat{T}_{y y}
\end{array}\right]
$$

and errors in the antenna reference frame translate into errors in the earth reference frame

$$
\left[\begin{array}{l}
\Delta \hat{T}_{h h} \\
\Delta \hat{T}_{v v}
\end{array}\right]=\frac{1}{A^{4}-B^{4}}\left[\begin{array}{rr}
A^{2} & -B^{2} \\
-B^{2} & A^{2}
\end{array}\right]\left[\begin{array}{l}
\Delta \hat{T}_{x x} \\
\Delta \hat{T}_{y y}
\end{array}\right] .
$$

Note that the errors tend to infinite wherever $A=B$, as studied in [8].

Errors in $\operatorname{Im}\left[\hat{T}_{y x}-\hat{T}_{y x}^{i d}\right]$ [see (8)] are specially important because of the phase difference between the co- and cross-polar antenna voltage patterns, and may ultimately limit the capabilities of using the measurement of the third Stokes parameter to correct for Faraday rotation effects ${ }^{2}$ [see (8)] [9], necessary to derive $T_{h h}$ and $T_{v v}$ accurately. ${ }^{3}$ Additionally, even though the measurement of $\hat{T}_{y x}$ allows to obtain $\hat{T}_{h h}$ and $\hat{T}_{v v}$ maps in the whole alias-free field of view without singularities [see (11); matrix in (3) is never singular], errors in $\hat{T}_{y x}$ directly translate into $\hat{T}_{h h}$ and $\hat{T}_{v v}$ through (12). The singularity problem can be avoided and cross-polar pattern errors minimized if the geophysical parameter retrieval algorithms are designed to operate with brightness temperature maps in the antenna reference frame-although Faraday rotation correction will still be necessary—or to operate on $\hat{I}=\hat{T}_{x x}+\hat{T}_{y y}=\hat{T}_{h h}+\hat{T}_{v v}$, which is invariant to rotations (see [11] for ocean salinity retrieval study). $\hat{I}$ can be computed as $\hat{I}=\hat{T}_{x x}+\hat{T}_{y y}$, where $\hat{T}_{x x}$ and $\hat{T}_{y y}$ are measured in the dual polarization mode.

\section{CONCLUSION}

This work has presented the extension of the formulation of the visibility function to include the cross-polar voltage patterns, which may be different from element to element. Its impact on the radiometric accuracy has been analyzed using SEPS and antenna patterns measured by EADS-CASA espacio for the first nine MIRAS receivers, showing a degradation smaller than $0.18 \mathrm{~K}$ root mean square within the alias-free field of view.

\footnotetext{
${ }^{2}$ The average daytime Faraday rotation at L-band is $17^{\circ} / f^{2}$ ( $f$ in gigahertz), which is $8.7^{\circ}$ approximately [10]. However, it can exhibit very large variations, depending on the local time, season, solar activity, and geographic location.

${ }^{3}$ The brightness temperature sensitivity to sea surface salinity is $0.5 \mathrm{~K} / \mathrm{psu}$ at $25{ }^{\circ} \mathrm{C}$, and the salinity retrieval accuracy is $0.1 \mathrm{psu}$ after spatio-temporal averaging in $200-\mathrm{km}$ boxes and 30 days.
} 


\section{REFERENCES}

[1] M. Martín-Neira and J. M. Goutoule, "MIRAS-A two-dimensional aperture-synthesis radiometer for soil-moisture and ocean salinity observations," ESA Bull., no. 92, pp. 95-104, Nov. 1997.

[2] P. Sivestrin, M. Berger, Y. Kerr, and J. Font, "ESA's second earth explorer opportunity mission: The Soil Moisture and Ocean Salinity mission-SMOS," IEEE Geosci. Remote Sens. Newslett., no. 118, pp. 11-14, 32001.

[3] I. Corbella, N. Duffo, M. Vall-1lossera, A. Camps, and F. Torres, "The visibility function in interferometric aperture synthesis radiometry," IEEE Trans. Geosci. Remote Sens., vol. 42, no. 8, pp. 1677-1682, Aug. 2004

[4] J. P. Claassen and A. K. Fung, "The recovery of polarized apparent temperature distributions of flat scenes from antenna temperature measurements," IEEE Trans. Antennas Propagat., vol. AP-22, pp. 433-442, 1974

[5] M. Martín-Neira, S. Ribó, and A. J. Martín-Polegre, "Polarimetric mode of MIRAS," IEEE Trans. Geosci. Remote Sens., vol. 40, no. 8, pp. 1755-1768, Aug. 2002.

[6] A. C. Ludwig, "The definition of cross polarization," IEEE Trans. Antennas Propagat., vol. AP-21, no. 1, pp. 116-119, Jan. 1973.
[7] A. Camps, I. Corbella, M. Vall-llossera, N. Duffo, F. Marcos, F Martínez-Fadrique, and M. Greiner, "The SMOS end-to-end performance simulator: Description and scientific applications," in Proc. IGARSS, vol. 1, Toulouse, France, Jul. 21-25, 2003, pp. 13-15.

[8] P. Waldteufel and G. Caudal, "About off-axis radiometric polarimetric measurements," IEEE Trans. Geosci. Remote Sens., vol. 40, no. 6, pp 1435-1439, Jun. 2002.

[9] S. H. Yueh, "Estimates of faraday rotation with passive microwave polarimetry for microwave remote sensing of earth surfaces," IEEE Trans. Geosci. Remote Sens., vol. 38, no. 9, pp. 2434-2438, Sep. 2000.

[10] J. P. Hollinger and R. C. Lo, "Low-frequency microwave radiometer for N-ROSS, in Large Space Antenna Sys. Technol.," in NASA Conf. Publ., vol. 2368, 1984, pp. 87-95.

[11] A. Camps, I. Corbella, M. Vall-llossera, N. Duffo, F. Torres, R. Villarino, L. Enrique, F. Julbé, J. Font, A. Julià, C. Gabarró, J. Etchetto, J. Boutin, A. Weill, V. Caselles, E. Rubio, P. Wursteisen, and M. MartínNeira, "L-Band sea surface emissivity: Preliminary results of the WISE2000 campaign and its application to salinity retrieval in the SMOS mission," Radio Sci., vol. 38, no. 4, pp. 8071-8071, Jun. 2003. DOI: 10.1029/2002RS002629. 